\title{
Some Remarks on the Uniformizing Function in Genus 2
}

\author{
PETER BUSER $^{1}$ and ROBERT SILHOL ${ }^{2}$ \\ ${ }^{1}$ Section de Mathématiques, IGAT, Geom, Station 8, Ecole Polytechnique Fédérale de \\ Lausanne, CH-1015 Lausanne, Switzerland. e-mail: peter.buser@epfl.ch \\ ${ }^{2}$ Département de Mathématiques, Université Montpellier II, UMR CNRS 5149, Place \\ E. Bataillon 34095 Montpellier Cedex 5, France. e-mail: rs@math.univ-montp2.fr
}

(Received: 30 September 2004; accepted in final form: 4 May 2005)

\begin{abstract}
This article describes a practical procedure to compute, for any Fuchsian group of genus 2 acting on the unit disc, a uniformizing function for the corresponding algebraic curve.
\end{abstract}

Mathematics Subject Classification (2000). 30Fxx.

Key words. Riemann surfaces, algebraic curves, numerical uniformization.

\section{Introduction}

In this paper we propose a numerical method for the uniformization of Riemann surfaces and algebraic curves in genus two with higly accurate results.

Let $G$ be a Fuchsian group acting on the unit disk $\mathbb{D}$, and let $S=\mathbb{D} / G$. As is well known $S$ is also in natural way an algebraic curve. We propose to describe a practical way to compute, in genus 2, the uniformizing function from the unit disk to the algebraic curve. By practical we mean that the method we are going to describe leads to a simple algorithm easy to implement that will compute an approximation of the uniformizing function.

The basic idea underlying our method is to reduce the problem to the known case of genus 1 (for which solutions to the uniformization problem are known since the XIXth century). Geometrically, a natural way to associate a genus 1 surface to a genus 2 surface is the following. Let $X$ be a genus 2 surface and let $Y$ be a genus 3 double cover. Let $\varphi$ be the involution such that $X=Y / \varphi$. By a classical result of Enriques such a genus 3 surface is hyperelliptic. Let $\tau$ be the hyperelliptic involution on $Y$. Then the quotient of $Y$ by $\tau \circ \varphi$ is of genus 1 . In practice we will work with a fundamental octagon representing the genus 2 surface $S$, take $G$ to be the group generated by the side paring of opposite sides, and produce from $G$ a group $G^{\prime}$ such that $S^{\prime}=\mathbb{D} / G^{\prime}$ is of genus 1 . This surface $S^{\prime}$ has also a natural representation as $\mathbb{C} / \Lambda$. From this we will show how to construct a holomorphic function $f: \mathbb{D} \rightarrow \mathbb{C}$ such that the composition $T_{\Lambda} \circ f$, of $f$ with a $\Lambda$-equivariant function $T_{\Lambda}$, is a $G$-equivariant meromorphic function. 
Fenchel-Nielsen coordinates are a more familiar way of parameterizing Teichmüller space (or if one prefers, hyperbolic surfaces). In a second part we will show how one can associate octagons in terms of Fenchel-Nielsen coordinates and vice-versa, using essentially algebraic relations.

Combining these two parts we obtain a way to produce a fairly simple algorithm to compute an approximation of uniformizing functions. This algorithm has been implemented, and in the third part we give a few examples of the results. The code in Maple form, and in $\mathrm{C}$ (with a Maple interface), together with a worksheet containing documentation and examples is available at http://www.math. univ-montp2.fr/ rs (see in particular [7]).

\section{From the Octagon to the Uniformizing Function}

Let $G$ be a Fuchsian group acting on the unit disk $\mathbb{D}$ and such that $\mathbb{D} / G=S$ is a genus 2 surface. We assume here that $G$ does not contain parabolic or elliptic elements. It is well known that under this condition one can choose a geodesic octagon for the fundamental domain that has a central symmetry and such that $G$ operates by identification of opposite sides. Conjugating $G$ in $\mathrm{PSL}_{2}(\mathbb{R})$, if necessary, we may assume in addition that the octagon is symmetric with respect to the origin and has the midpoint of one of its sides on the real axis.

Let us now assume that such an octagon, $\mathscr{P}$, is given. We label the vertices $q_{1}, \ldots, q_{8}$ and the midpoints of the sides $p_{1}, \ldots, p_{8}$ as in Figure 1. The preimages of the Weierstrass points of $S$ are 0 , the $p_{i}$ 's and the $q_{i}$ 's. Call $g_{i}$ the element of $G$ such that $g_{i}\left(p_{i}+4\right)=p_{i}$. Then $G$ is generated by $\left\{g_{1}, \ldots, g_{4}\right\}$.

The uniformizing function for the corresponding algebraic curve will be obtained from this data.

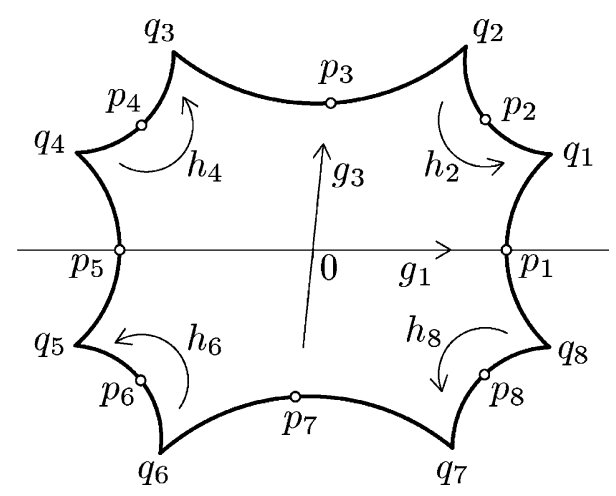

Figure 1.

As is well known an elliptic element of $\operatorname{Aut}(\mathbb{D})$ of a given order is completely determined by its fixed point in $\mathbb{D}$. We will denote by $h_{2 i}$ the elliptic transfor- 
mations of order 2 with fixed points $p_{2 i}$ and call $G^{\prime}$ the group generated by $\left\{g_{1}, g_{3}, h_{2}, h_{4}, h_{6}, h_{8}\right\}$. The crucial observation is that $\mathscr{P}$ is also a fundamental domain for the action of $G^{\prime}$ and, moreover, for each $g^{\prime} \in G^{\prime}$ there exists a $g \in G$ such that either $g^{\prime}(z)=g(z)$ or $g^{\prime}(z)=g(-z)$.

The surface $S^{\prime}=\mathbb{D} / G^{\prime}$ is a surface of genus 1 with four cone points, the images of the $p_{2 i}$ 's.

The key to our construction is contained in the next Lemma.

LEMMA 1.1. In the above situation there exists a unique $\tau$ in the upper half plane $\mathbb{H}$ and a unique holomorphic function $f: \mathbb{D} \rightarrow \mathbb{C}$ such that

(i) $f$ is odd;

(ii) $f$ is conformal on $\mathbb{D}$ except at the points $p_{2 i}$ and their images under $G$ (or $\left.G^{\prime}\right)$ and $f(z)-f\left(p_{2 i}\right)$ is exactly of order 2 at $p_{2 i}$;

(iii) $f\left(g_{1}(z)\right)=2+f(z)$ and $f\left(g_{3}(z)\right)=2 \tau+f(z)$;

(iv) $f\left(h_{2 i}(z)\right)=f(z)$ for $i$ from 1 to 4 .

Proof. From a conformal point of view $S^{\prime}$ is a genus 1 surface with a distinguished homology basis defined by the images of the axes of $g_{1}$ and $g_{3}$. Hence there is a unique $\tau$ such that we have a conformal equivalence from $S^{\prime}$ to $\mathbb{C} / \Lambda$, where $\Lambda$ is the lattice generated by 2 and $2 \tau$, and the image of $[-1,1]$ (resp. $[-\tau, \tau])$ under the canonical projection $\pi_{2}: \mathbb{C} \rightarrow \mathbb{C} / \Lambda=S^{\prime}$ is in the same homology class as the image of the geodesic arc $\left[p_{5}, p_{1}\right]$ (resp. $\left[p_{7}, p_{3}\right]$ ). Note that these arcs are on the geodesic axes of $g_{1}$ and $g_{3}$ respectively. The conditions define the equivalence up to a translation in $\mathbb{C} / \Lambda$. We make it unique by requiring that the intersection of the axes maps to $\pi_{2}(0)$ and write $S^{\prime}=\mathbb{C} / \Lambda$.

Call $\pi_{1}$ the covering map $\mathbb{D} \rightarrow S^{\prime}$. Since $\pi_{2}: \mathbb{C} \rightarrow S^{\prime}$ is the universal cover of $S^{\prime}$ and $\mathbb{D}$ is simply connected, $\pi_{1}$ lifts to a map $f: \mathbb{D} \rightarrow \mathbb{C}$, and this lifting is unique if we impose $f(0)=0$.

It is readily checked that multiplication by -1 in $\mathbb{D}$ induces, via $\pi_{1}$, a non-trivial involution on $S^{\prime}$. Since $\pi_{1}(0)=\pi_{2}(0)$ is fixed by this involution it is also induced, via $\pi_{2}$, by multiplication by -1 in $\mathbb{C}$. Since by construction we have $\pi_{2} \circ f=\pi_{1}$, this proves that $f$ is odd.

Since $\pi_{2}$ is localy biholomorphic and $\pi_{1}$ is locally biholomorphic except at the $p_{2 i}$ 's and their images under $G$ (or $G^{\prime}$ ) where it is 2 to $1, f$ satifies (ii).

By construction there exists $\gamma_{1}$ and $\gamma_{3}$ in $\Lambda$ such that $f\left(g_{1}(z)\right)=f(z)+\gamma_{1}$ and $f\left(g_{3}(z)\right)=f(z)+\gamma_{3}$. To compute these we first note that $\pi_{1}\left(\left[p_{5}, p_{1}\right]\right)$ is a simple closed curve $\alpha$ in $S^{\prime}$. In the same way the image of $\left[p_{7}, p_{3}\right]$ is a simple closed curve $\beta$. Both $\alpha$ and $\beta$ have a natural orientation induced by the orientations of the intervals. On the other hand we have a canonical identification $\Lambda \cong H_{1}\left(S^{\prime}, \mathbb{Z}\right)$, and by definition of $\Lambda$ the homology class of $\alpha$ is identified with 2 and $\beta$ with $2 \tau$. In other words, $\gamma_{1}=2$ and $\gamma_{3}=2 \tau$. Hence $f$ satisfies (iii).

Again, for each $i$ there exist a $\gamma \in \Lambda$ such that $f\left(h_{2 i}(z)\right)=f(z)+\gamma$. Applying this at the fixed point $p_{2 i}$ we find $\gamma=0$. Hence, $f$ satisfies (iv). 
Note the following important consequences of the properties of $f$. Since $p_{5}=$ $-p_{1}$ and $p_{1}=g_{1}\left(p_{5}\right)$ we have $f\left(p_{1}\right)=1$. In the same way we have $f\left(p_{3}\right)=\tau$. We also have $f\left(q_{1}\right)=f\left(q_{2}\right), f\left(q_{3}\right)=f\left(q_{4}\right)$ and so forth. On the other hand $q_{1}=g_{1}\left(q_{4}\right)$ and $q_{3}=g_{3}\left(q_{6}\right)=g_{3}\left(-q_{2}\right)$. Combining these relations we find $f\left(q_{1}\right)=f\left(q_{2}\right)=1+\tau$, $f\left(q_{3}\right)=f\left(q_{4}\right)=-1+\tau$.

Figure 2 gives an illustration of how the map $f$ of the Lemma maps an octagon. To compute the function $f$ we will use the following simpler characterization

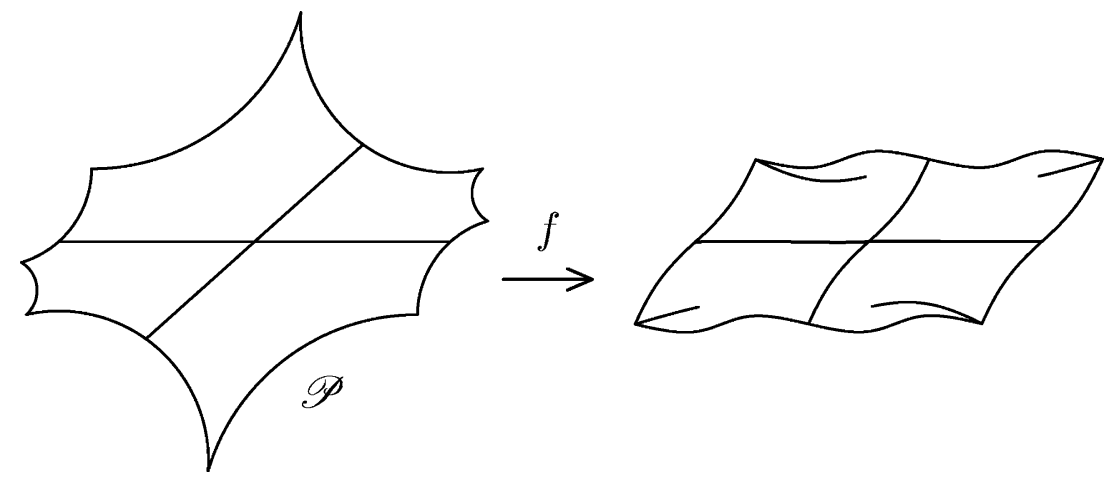

Figure 2.

LEMMA 1.2. Let notations be as above and let $\tilde{f}$ be a holomorphic function defined on a neighbourhood $U$ of the octagon $\mathscr{P}$. If there exists a $\tilde{\tau} \in \mathbb{C}$ such that the following hold,

(i) $\tilde{f}$ is odd,

(ii) $\tilde{f}\left(g_{1}(z)\right)=2+\tilde{f}(z)$ for points on the geodesic arc $\left[q_{4}, q_{5}\right]$,

(iii) $\tilde{f}\left(g_{3}(z)\right)=2 \tilde{\tau}+\tilde{f}(z)$ for points on the geodesic arc $\left[q_{6}, q_{7}\right]$,

(iv) $\tilde{f}\left(h_{2 i}(z)\right)=\tilde{f}(z)$ on $\left[q_{2 i-1}, q_{2 i}\right]$ for $i$ equal 1 and 2 ,

then $\tilde{f}=f$ on $U$, where $f$ is the function defined in Lemma 1.1.

Proof. Let $\tilde{\Lambda}$ be the lattice generated by 2 and $2 \tilde{\tau}$. Let $\tilde{S}^{\prime}=\mathbb{C} / \tilde{\Lambda}$ and $\tilde{\pi}_{2}$ be the canonical projection from $\mathbb{C}$ to $\tilde{S}^{\prime}$. Finally let $\tilde{\varphi}=\tilde{\pi}_{2} \circ \tilde{f}$ and $\varphi=\pi_{2} \circ f$, where $\pi_{2}$ is as in the proof of Lemma 1.1.

From the conditions satisfied by $f$ and $\tilde{f}$ it easily follows that $\tilde{\varphi} \circ \varphi^{-1}$ extends to a holomorphic map from $S^{\prime}$ to $\tilde{S}^{\prime}$ that sends $\pi_{2}(0)$ to $\tilde{\pi}_{2}(0)$. By standard elliptic curve theory this implies that $\tilde{\varphi} \circ \varphi^{-1}$ is induced by a map $z \mapsto a z$ in $\mathbb{C}$ (see for example [8] p. 159). But this implies that $\tilde{f}=a f$ for some complex number $a$. Since the conditions on $\tilde{f}$ imply, in the same way as for $f$, that $\tilde{f}\left(p_{1}\right)=1=f\left(p_{1}\right)$ we find $a=1$ and $\tilde{f}=f$. 
We can now give the construction of the $G$-equivariant function.

By standard elliptic function theory, we know that there exists an even meromorphic function $T_{\tau}: \mathbb{C} \rightarrow \mathbb{P}^{1}(\mathbb{C})$ such that $T_{\tau}$ is $\Lambda$-periodic, i.e., $T_{\tau}(z+2)=$ $T_{\tau}(z), T_{\tau}(z+2 \tau)=T_{\tau}(z)$. Moreover we can normalize it so that $T_{\tau}(0)=0, T_{\tau}(1)=1$ and $T_{\tau}(\tau)=\infty$. Explicitly we use the function,

$$
T_{\tau}(z)=-\frac{w}{K} \prod_{k=0}^{\infty} \frac{\left(w-\zeta^{2 k}\right)^{2}\left(1-\zeta^{2 k+2} w\right)^{2}}{\left(w-\zeta^{2 k+1}\right)^{2}\left(1-\zeta^{2 k+1} w\right)^{2}},
$$

where

$$
\zeta=\exp (\pi i \tau), \quad w=\exp (\pi i z) \quad \text { and } \quad K=4 \prod_{k=1}^{\infty}\left(\frac{1+\zeta^{2 k}}{1+\zeta^{2 k-1}}\right)^{4},
$$

(we have adapted for our purpose the function defined in Nehari [5], Chap. VI, Section 3).

PROPOSITION 1.4. Let, as in the beginning of this section, $G$ be a Fuchsian group identifying opposite sides of the octagon $\mathscr{P}$ and let $S=\mathbb{D} / G$. Let $f$ and $\tau$ be as Lemma 1.1 and finally let $F=T_{\tau} \circ f\left(T_{\tau}\right.$ as above). Then,

- For all $g \in G, F(g(z))=F(z)$.

- If $a_{1}=F\left(q_{i}\right), a_{2}=F\left(p_{2}\right)$ and $a_{3}=F\left(p_{4}\right)$ then

$$
y^{2}=x(x-1)\left(x-a_{1}\right)\left(x-a_{2}\right)\left(x-a_{3}\right)
$$

is an equation for the algebraic curve coresponding to $S$.

Proof. By the construction of $f$ we have $F\left(g_{1}(z)\right)=F(z)$ and $F\left(g_{3}(z)\right)=$ $F(z)$. So to prove the first point we only need to prove that $F\left(g_{2}(z)\right)=F(z)$ and $F\left(g_{4}(z)\right)=F(z)$. For this we note that $g_{2}(z)=-h_{6}(z)$. Hence, $f\left(g_{2}(z)\right)=$ $f\left(-h_{6}(z)\right)=-f(z)$ by the construction of $f$. Since $T_{\tau}$ is even we are done. The proof for $g_{4}$ is the same.

For the second point, recall that

$$
f(0)=0, \quad f\left(p_{1}\right)=1, \quad f\left(q_{1}\right)=1+\tau \quad \text { and } \quad f\left(p_{3}\right)=\tau .
$$

Now note that $F$ is precisely branched over $\infty, 0,1, a_{1}$ (the branch points of $T_{\tau}$ ), $a_{2}$ and $a_{3}$ (these induced by $f$ ). Since $S$ is of genus 2 , and in particular hyperelliptic, this implies that $F$, which is $G$-equivariant, is a lifting of the hyperelliptic projection $S \rightarrow \mathbb{P}^{1}(\mathbb{C})$. The assertion then follows from the fact that $\infty, 0,1$ and the $a_{i}$ 's are the images of the Weierstrass points.

If we write the equation of the algebraic curve $C$ in the form of $y^{2}=$ $x(x-1)\left(x-a_{1}\right)\left(x-a_{2}\right)\left(x-a_{3}\right)$, then the function $F(z)$ will be the $x$ coordinate of the corresponding point in $C$. Now $F$ is an even function, and hence the same holds for $P_{S}: z \mapsto F(z)(F(z)-1)\left(F(z)-a_{1}\right)\left(F(z)-a_{2}\right)\left(F(z)-a_{3}\right)$. Since $P_{S}$ has also a zero of 
order 2 at the origin, $P_{S}$ has a development at the origin of the form $\sum a_{n} z^{2 n}$ (with $a_{1} \neq 0$ ). From this it is a trivial matter to define a meromorphic square root of $P_{S}$ and hence a $G$-equivariant function giving the $y$ coordinate.

Remark 1.6. For technical reasons we have used the function $T_{\tau}$, but we could have used to the same effect any even $\Lambda$-periodic function. In particular we could have used the Weierstrass $\wp$ function.

The numerical part in our approach is the computation of the function $f$. Here the essential point is that $f$ maps $\mathscr{P}$ to a bounded domain. This allows as to use polynomial approximation (cf. points 3.2, 3.3 for details).

\section{From Fenchel-Nielsen Coordinates to Octagons}

In this section we relate the octagon $\mathscr{P}$ to the Fenchel-Nielsen coordinates which are geometrically more intuitive. These parameters arize when a Riemann surface is partitioned into pairs of pants, i.e. surfaces of signature $(0,3)$ with geodesic boundary curves. In genus 2 , the number of pairs of pants is two, and there are two combinatorially different partition schemes. We take here the one in which the partition is along (pairwise disjoint simple closed) geodesics $\gamma_{1}, \gamma_{2}, \gamma_{3}$, each of which is nonseparating. There are many ways to adapt such a partition to $\mathscr{P}$, we describe here two possibilities which have proved useful for the computations of the examples in Section 3. These two decompositions are illustrated in Figures 3 and 4 . We will describe in detail one of these (Figure 3) and only sketch the second since the method is essentially the same.

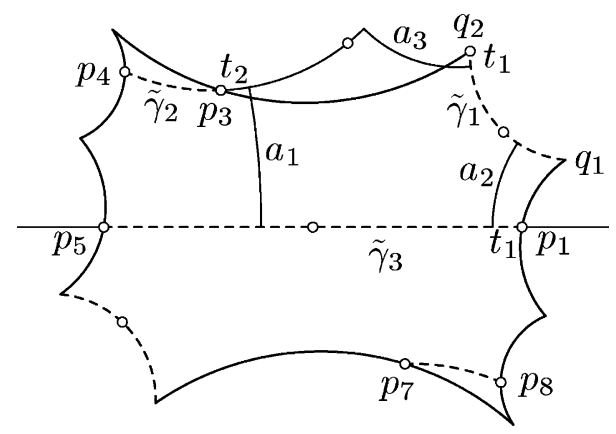

Figure 3.

Consider the following geodesics in $\mathbb{D}: \tilde{\gamma}_{1}$ through $q_{1}, q_{2} ; \tilde{\gamma}_{2}$ through $p_{3}, p_{4} ; \tilde{\gamma}_{3}$ through $p_{5}, p_{1}$. On $S$ the geodesics $\gamma_{i}=\pi\left(\tilde{\gamma}_{i}\right), i=1,2,3$, then form a partition of the required type. Note that the inverse image of $\gamma_{2}$ in $\mathscr{P}$ is the union of the arcs $p_{3} p_{4}$ and $p_{7} p_{8}$. 
The length parameters of this partition are defined as

$$
l_{i}=\frac{1}{2} \ell\left(\gamma_{i}\right), \quad i=1,2,3 .
$$

Observe that

$$
l_{1}=\operatorname{dist}\left(q_{1}, q_{2}\right) / 2, \quad l_{2}=\operatorname{dist}\left(p_{3}, p_{4}\right), \quad l_{3}=\operatorname{dist}\left(0, p_{1}\right) .
$$

For the twist parameters we draw, for $i=1,2,3$, the shortest connection $a_{i}$ in $\mathbb{D}$ from $\tilde{\gamma}_{i-1}$ to $\tilde{\gamma}_{i+1}$ (indices modulo 3 ) to obtain a right angled geodesic hexagon $H$ with sides $a_{1}, \gamma_{3}^{H}, a_{2}, \gamma_{1}^{H}, a_{3}, \gamma_{2}^{H}$. Under $\pi$ this hexagon projects to a hexagon on one of the pairs of pants of the partition, and it is known that

$$
\ell\left(\gamma_{i}^{H}\right)=l_{i}, \quad i=1,2,3 .
$$

The lengths of the $a_{i}$ are given by

$$
\cosh a_{k}=\frac{\cosh l_{k}+\cosh l_{k-1} \cosh l_{k+1}}{\sinh l_{k-1} \sinh l_{k+1}}, \quad k=1,2,3 \bmod 3 .
$$

On $\tilde{\gamma}_{1}$ there is an arc $t_{1}$ connecting the end point of $a_{3}$ with $q_{2}$. We denote by $\ell_{o r}\left(t_{1}\right)$ its oriented length: positive if an observer walking along $a_{3}$ towards $\tilde{\gamma}_{1}$ has to turn left in order to get to $q_{2}$ (as in Figure 3), negative otherwise. There are similar such arcs $t_{2}$ on $\tilde{\gamma}_{2}$ from $a_{1}$ to $p_{3}$ and $t_{3}$ on $\tilde{\gamma}_{3}$ from $a_{2}$ to $p_{1}$. The twist parameters are now defined as

$$
\tau_{i}=\frac{\ell_{\text {or }}\left(t_{i}\right)}{l_{i}}, \quad i=1,2,3 .
$$

The computation of the Fenchel-Nielsen parameters thus defined is based on Möbius transformations. Let $\mathscr{M}$ be the algebra of all $2 \times 2$ matrices $M$ of type

$$
M=\left[\begin{array}{ll}
\alpha & \beta \\
\bar{\beta} & \bar{\alpha}
\end{array}\right], \quad \alpha, \beta \in \mathbb{C}, \quad \operatorname{det} M \neq 0 .
$$

Any $M \in \mathscr{M}$ acts on $\mathbb{D}$ as an isometry, sending points $z \in \mathbb{D}$ to

$$
M[z]=\frac{\alpha z+\beta}{\bar{\beta} z+\bar{\alpha}}, \quad \text { if det } M>0, \quad M[z]=\frac{\alpha / \bar{z}+\beta}{\bar{\beta} / \bar{z}+\bar{\alpha}}, \quad \text { if } \operatorname{det} M<0 .
$$

The isometry is orientation preserving if det $M>0$, and orientation reversing otherwise.

Explicitly we shall use, for any $p \in \mathbb{D}$ the matrix

$$
H(p)=i\left[\begin{array}{ll}
\left(1+|p|^{2}\right) & -2 p \\
2 \bar{p} & -\left(1+|p|^{2}\right)
\end{array}\right]
$$

and for $t \in \mathbb{R}$ the matrices

$$
R(t)=\left[\begin{array}{ll}
1+\cosh t & \sinh t \\
\sinh t & 1+\cosh t
\end{array}\right], \quad U(t)=\left[\begin{array}{ll}
1+\cosh t & i \sinh t \\
-i \sinh t & 1+\cosh t
\end{array}\right] .
$$


$H(p)$ represents the half turn $h_{p}$ with center of rotation $p ; R(t)$ is the hyperbolic isometry with axis from -1 to 1 , shifting points on this axis to the right or to the left by $|t|$ according as $t>0$ or $t<0 ; U(t)$ has similar properties with axis from $-i$ to $i$, shifting points on the axis upwards if $t>0$ and downwards if $t<0$.

Using Figure 3, one gets the following expressions for $p_{1}, \ldots, p_{4}, q_{2}$ in terms of Fenchel-Nielsen coordinates, where the $a_{k}$ are given by (2.4).

$$
\begin{aligned}
& p_{1}=R\left(l_{3}\right)[0], \\
& p_{2}=R\left(l_{3}-\tau_{3} l_{3}\right) U\left(a_{2}\right) R\left(-\tau_{1} l_{1}\right)[0], \\
& p_{3}=R\left(-\tau_{3} l_{3}\right) U\left(a_{1}\right) R\left(-\tau_{2} l_{2}\right)[0], \\
& p_{4}=R\left(-\tau_{3} l_{3}\right) U\left(a_{1}\right) R\left(-l_{2}-\tau_{2} l_{2}\right)[0], \\
& q_{2}=R\left(l_{3}-\tau_{3} l_{3}\right) U\left(a_{2}\right) R\left(-l_{1}-\tau_{1} l_{1}\right)[0] .
\end{aligned}
$$

The vertices of the octagon are obtained via the half turns,

$$
q_{3}=H\left(p_{3}\right)\left[q_{2}\right], \ldots, q_{1}=H\left(p_{1}\right)\left[q_{8}\right] .
$$

For the computation in the inverse direction we define the geometric trace

$$
\operatorname{gtr} M=\frac{1}{2} \frac{\operatorname{trace} M}{\sqrt{|\operatorname{det} M|}}
$$

for $M \in \mathscr{M}$, and the traceless part

$$
\operatorname{tl}(M)=M-\frac{1}{2} \operatorname{trace} M \cdot\left[\begin{array}{ll}
1 & 0 \\
0 & 1
\end{array}\right] .
$$

The following properties are easily checked using conjugation into a suitable normal form.

- If $M$ is a rotation with center of rotation $p \in \mathbb{D}$, then $\operatorname{tl}(M)$ is the half turn with center $p$.

- If $M$ is an isometry (orientation preserving or reversing) having an axis $\gamma$, then $\operatorname{tl}(M)$ is the symmetry with axis $\gamma$.

- If $L, L^{\prime}$ are half turns with centers of rotation $p, p^{\prime}$, then

$$
\left|\operatorname{gtr} L L^{\prime}\right|=\cosh \left(\operatorname{dist}\left(p, p^{\prime}\right)\right) .
$$

- If $L$ is a half turn with center of rotation $p$ and $S$ a symmetry with axis $\gamma$, then $\operatorname{gtr} L S= \pm \sinh (\operatorname{dist}(p, \gamma))$ with the plus sign on one side of $\gamma$ and the minus sign on the other.

Let us now assume that the octagon $\mathscr{P}$ is given, say in terms of the vertices $q_{1}, \ldots, q_{8}$. Using the formulae

$$
p=\frac{\omega}{1+\sqrt{1-|\omega|^{2}}}, \quad \text { with } \omega=\frac{q\left(1-\left|q^{\prime}\right|^{2}\right)+q^{\prime}\left(1-|q|^{2}\right)}{1-\left|q q^{\prime}\right|^{2}}
$$


for the midpoint $p$ of a geodesic segment $q q^{\prime}$ in $\mathbb{D}$ we obtain the midpoints of the sides. Abbreviating

$$
\begin{aligned}
& M_{1}=H\left(q_{2}\right) H\left(p_{2}\right), \quad M_{2}=H\left(p_{4}\right) H\left(p_{3}\right), \quad M_{3}=H\left(p_{1}\right) H(0), \\
& L_{1}=H\left(q_{2}\right), \quad L_{2}=H\left(p_{3}\right), \quad L_{3}=H\left(p_{1}\right),
\end{aligned}
$$

and using (2.2) we then obtain the following expressions for the Fenchel-Nielsen coordinates

$$
\begin{aligned}
\cosh l_{i} & =\left|\operatorname{gtr} M_{i}\right|, \\
\sinh \tau_{i} l_{i} & =\operatorname{gtr}\left(L_{i} \operatorname{tl}\left(\operatorname{tl}\left(M_{i+1}\right) \operatorname{tl}\left(M_{i}\right)\right)\right), \quad i=1,2,3,
\end{aligned}
$$

(indices modulo 3). Here it is used that the product of the symmetries $\operatorname{tl}\left(M_{i+1}\right)$ and $\operatorname{tl}\left(M_{i}\right)$ is a hyperbolic isometry, whose axis is the common orthogonal of the axes of $M_{i+1}$ and $M_{i}$. In order to see that the signs are correct it suffices, by continuity, to check this for a convenient special case.

Figure 4 shows, by way of an example, how one may obtain other octagons for the same group $G$ (up to conjugation). The figure on the right-hand side shows our second decomposition that we used for the computations in Section 3.
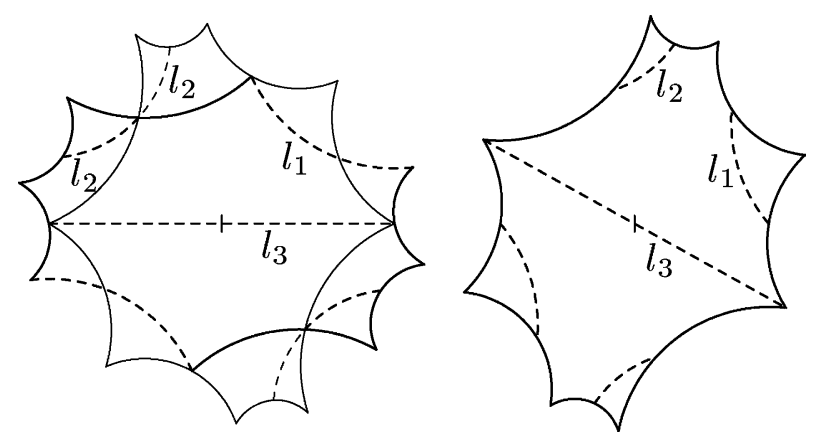

Figure 4

In a first step we set

$$
\begin{aligned}
& p_{1}^{\prime}=p_{2}, p_{2}^{\prime}=q_{2}, p_{3}^{\prime}=H\left(p_{3}\right)\left[p_{4}\right], p_{4}^{\prime}=p_{3} \\
& q_{1}^{\prime}=H\left(p_{2}\right)\left[p_{1}\right], q_{2}^{\prime}=H\left(p_{2}^{\prime}\right)\left[q_{1}^{\prime}\right], q_{3}^{\prime}=H\left(p_{3}\right)\left[p_{5}\right], q_{4}^{\prime}=p_{5},
\end{aligned}
$$

and $p_{k+4}^{\prime}=-p_{k}^{\prime}, q_{k+4}^{\prime}=-q_{k}^{\prime}$, for the remaining points. It is easily checked that the octagon $\mathscr{P}^{\prime}$ with these data is also a fundamental domain of $G$.

In the second step we rotate $\mathscr{P}^{\prime}$ so that the midpoint of the first side lies on the real axis. The formulas for $p_{1}^{\prime}, \ldots, p_{4}^{\prime}, q_{8}^{\prime}$ are 


$$
\begin{aligned}
& p_{1}^{\prime}=R\left(l_{3}-\tau_{3} l_{3}\right) U\left(a_{2}\right) R\left(-\tau_{1} l_{1}\right)[0], \\
& p_{2}^{\prime}=R\left(l_{3}-\tau_{3} l_{3}\right) U\left(a_{2}\right) R\left(-\tau_{1} l_{1}-l_{1}\right)[0], \\
& p_{3}^{\prime}=R\left(-\tau_{3} l_{3}\right) U\left(a_{1}\right) R\left(l_{2}-\tau_{2} l_{2}\right)[0], \\
& p_{4}^{\prime}=R\left(-\tau_{3} l_{3}\right) U\left(a_{1}\right) R\left(-\tau_{2} l_{2}\right)[0], \\
& q_{8}^{\prime}=R\left(l_{3}\right)[0]
\end{aligned}
$$

The remaining vertices are $q_{1}^{\prime}=H\left(p_{1}^{\prime}\right)\left[q_{8}^{\prime}\right]$, etc. and the resulting octagon has to be rotated by multiplying with $\left|p_{1}^{\prime}\right| / p_{1}^{\prime}$.

\section{The Implementation and Some Examples}

It is fairly straightforward to go from the method we have indicated in the first two sections to an implementation. We have done this in the form of a Maple package that can be obtained at [http://www.math.univ-montp2.fr/ rs].

We describe briefly the content of the file genus2unif.mp (the Maple package). Details on its usage are given in the files genus2unifdoc.mws (a Maple worksheet, see [7] for an html version).

The essential functions available are

\subsection{OCTO1 AND OCTO2}

These compute, using the formulae given in Section 2, the vertices and midpoints of a fundamental octagon in terms of the Fenchel-Nielsen coordinates of the hyperbolic surface. The pants decomposition will be as on the left of Figure 5 for Octo1 and as on the right for Octo2.
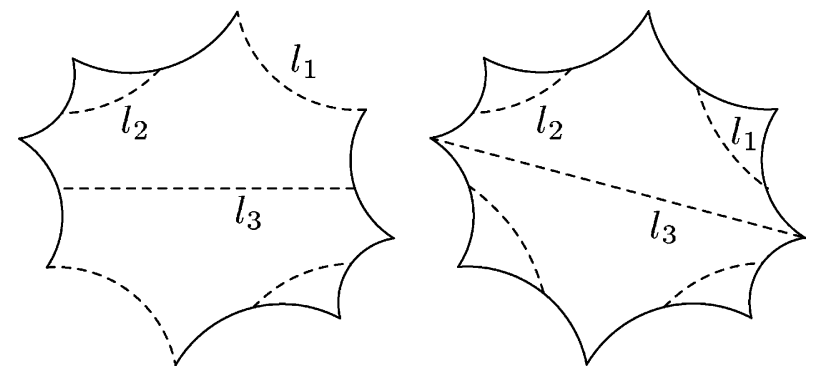

Figure 5.

\subsection{G2UNIFCOEFF}

This computes the coefficients of a polynomial approximation $\sum_{n=1}^{N} a_{n} z^{2 n-1}$ (where $N$ can be chosen) of the holomorphic function $f$, described in Lemma 1.1, 
in terms of the octagon. For this we construct an over-determined linear system where the unknowns are $\tau$ and the coefficients $a_{n}$. The coefficients of the system are the conditions of Lemma 1.2 at points on the boundary of the octagon. We then solve this system in the sense of least squares using standard routines to do this. The reason this works, and in fact works rather well, is that the function $f$ we are approximating is holomorphic on the whole unit disk so that already the Taylor polynomials provide arbitrarily accurate approximations.

Remark 3.3. What G2unifcoeff does then is to find, for a given degree, the best possible polynomial approximation of $f$ on the octagon and not to compute an approximation of the Taylor expansion. It turns out experimentally, however that roughly the first quarter to the first third of the coefficients are rather good approximations of the Taylor coefficients.

\subsection{G2UNIFFUNC}

This is the composition of the approximation of the function $f$, computed by means of 3.2, and the inverse elliptic function described in 1.3. It is an approximation of an even $G$-invariant function on the unit disk (see 1.4).

\subsection{G2EQUA}

This evaluates the function computed in 3.3 at the Weierstrass points. The output is a list of 5 complex numbers: 0 , the image of $0 ; 1$, the image of $p_{1}$ (in the notation of Figure 1); $a_{1}$, the image of $q_{1}$ (or of any $q_{i}$ ); $a_{2}$, the image of $p_{2}$; and $a_{3}$, the image of $p_{4}$. The remaining Weierstrass point $p_{3}$ is sent to infinity by this map.

Remark 3.6. We also give variants for the case of surfaces with an order 4 automorphism. Taking into account the additional symmetry allows us to speed up the procedure somewhat.

We have also added a few further routines which may be useful (see the file genus2unifdoc.mws or [7] for more detail).

A great number of tests has been carried out. These contain, of course, the known cases of surfaces with a very large automorphism group, that is the surfaces with Fenchel-Nielsen coordinates:

$-\left(1+\sqrt{2}, 0,1+\sqrt{2}, 0,3+2 \sqrt{2}, \frac{1}{2}\right)$ (where as before $1+\sqrt{2}$ and $3+2 \sqrt{2}$ refer to the hyperbolic cosine of half the length of the geodesic, and 0 and $\frac{1}{2}$ are the corresponding twist parameters). This is the surface with automorphism group of order 48.

- $(2,0,2,0,2,0)$. This is the surface with automorphism group of order 24 . We have also made the computations with the coordinates $\left(2, \frac{1}{2}, 2, \frac{1}{2}, 5, \frac{1}{2}\right)$. 
- $\left(3 / 2+\sqrt{5} / 2,0,1+\sqrt{5} / 2, \frac{1}{2}, 9 / 4+3 \sqrt{5} / 4, \frac{1}{2}\right)$. This is the surface with automorphism group of order 10 .

The results are the ones expected within the accuracy demanded (see [7] for more details).

We have also tested the method on the exact examples computed in [3]. The tests for a few of these are given in [7] but we have favored examples for which we have no proof of exactness. These are the surfaces with Fenchel-Nielsen coordinates:

- $\left(2, \frac{1}{2}, 4, \frac{1}{2}, 7, \frac{1}{2}\right)$ (which is the same surface as the one with Fenchel-Nielsen coordinates $(\sqrt{2}, 0, \sqrt{2}, 0,2,0)$ of [3] 8.6).

$-(\sqrt{7}, 0, \sqrt{7}, 0,2,0)$ of [3] 8.7 .

- $\left(3 / 2, \frac{1}{2}, 3 / 2, \frac{1}{2}, 4, \frac{1}{2}\right)$ (which is the same surface as the one with coordinates $(\sqrt{6}, 0, \sqrt{6}, 0,3 / 2,0)$ of $[3] 8.8)$.

$-(3,0,3,0,3,0)$ of [3] 8.9 .

The example $(2,0,4,0,7,0)$ comes from $[6]$ and $(4,0, \sqrt{10} / 2,0, \sqrt{10}, 0)$ comes from [1]. The results are the same as the ones announced there. The interest of these examples is that they have the hyperelliptic involution as only non-trivial automorphism.

The other examples are to our knowledge new. For the first three one can in fact prove that the results are exact, but this will be done elsewhere.

$-\left(1+\sqrt{3}, \frac{1}{4}, 1+\sqrt{3}, \frac{1}{4}, 3+2 \sqrt{3}, \frac{1}{2}\right)$. For this example we find, with 25 exact digits, the equation

$$
y^{2}=x\left(x^{2}-1\right)(x-(\sqrt{3}+i) / 2)(x-(\sqrt{3}-i) / 2) .
$$

$-\left(1+\sqrt{3}, \frac{1}{4}, 3+2 \sqrt{3}, 0,1+\sqrt{3}, \frac{1}{4}\right)$. For this example we find the equation

$$
y^{2}=\left(x^{2}-(2-\sqrt{3}) i\right)\left(x^{2}-1\right)\left(x^{2}-1-(2-\sqrt{3}) i\right) .
$$

The main interest of this example is that the algebraic curve is not defined over $\mathbb{R}$ (this can be checked by computing the Igusa invariants - see [7]).

- $\left((1+\sqrt{17}) / 4, \frac{1}{2},(1+\sqrt{17}) / 4, \frac{1}{2},(3+\sqrt{17}) / 2, \frac{1}{2}\right)$. Here we find the equation

$$
y^{2}=x\left(x^{2}-1\right)(x-(31-7 \sqrt{17}) / 64)(x-(31+7 \sqrt{17}) / 2) .
$$

$-((1+\sqrt{5}) / 2,0,(1+\sqrt{5}) / 2,0,(1+\sqrt{5}) / 2,0)$. We find that an equation should be

$$
y^{2}=x(x-1)(x-(5+\sqrt{5}) / 2)(x-1-2 \sqrt{5} / 5)(x-6-2 \sqrt{5}) .
$$

$-\left(3, \frac{1}{2}, 3, \frac{1}{2}, 3, \frac{1}{2}\right)$ for which we find that an equation should be

$$
y^{2}=x(x-1)(x-2 / 5+4 i / 5)(x-3 / 5+4 i / 5)\left(x-\frac{1}{2}+i\right) .
$$

The second form $\left(2, \frac{1}{2}, 2, \frac{1}{2}, 3,0\right)$ of this surface is also tested. 
$-\left(3+2 \sqrt{2}, 0,3+2 \sqrt{2}, \frac{1}{2}, 3+2 \sqrt{2}, \frac{1}{2}\right)$ and $\left(1+\sqrt{2}, 0,1+\sqrt{2}, 0,1+\sqrt{2}, \frac{1}{2}\right)$. The equations found are rather complicated and it is not very meaningful to give them here. On the other hand, the results we find suggest that the first is isomorphic to the algebraic curve defined by the equation,

$$
y^{2}=\left(x^{2}-1\right)\left(x^{4}+(128-90 \sqrt{2}) x^{2}+498-352 \sqrt{2}\right),
$$

while the second would be isomorphic to the one defined by

$$
y^{2}=\left(x^{2}-1\right)\left(x^{4}+(128+90 \sqrt{2}) x^{2}+498+352 \sqrt{2}\right) .
$$

As for other examples the exact forms we suggest here come from comparisons with computations made with other Fenchel-Nielsen coordinates and corroborations with computations made on transforms (those indicated in [3]). These examples are in fact 8.2 and 8.3 of [4] and hence the Fuchsian groups are arithmetic.

- The description of our last example is involved. Let $L=5 / 2$ and let

$$
t w=\operatorname{arccosh}\left(\sqrt{\frac{(L-1)(L+1)}{2 L}}\right) / \operatorname{arccosh}(L)
$$

(this value of the twist parameter is to insure that the algebraic curve is in a certain family of real curves). Then for the surface with Fenchel-Nielsen coordinates $(L, t w, L, t w, 2 L+1,1 / 2)$ we find the equation,

$$
y^{2}=x^{5}-\frac{13 \sqrt{2}}{16} x^{4}+\frac{13 \sqrt{2}}{16} x^{2}-x .
$$

\section{Acknowledgements}

Partially supported by the Swiss National Science Foundation, Contract 20-68181.02. Many hearty thanks to Aline Aigon, Hugo Akrout and Philipe Elbaz-Vinent for interesting discussions and important comments and suggestions.

\section{References}

1. Aigon, A. and Silhol, R.: Hyperbolic hexagons and algebraic curves in genus 3, J. London Math. Soc. 66 (2002), 671-690.

2. Buser, P.: Geometry and Spectra of Compact Riemann Surfaces, Birkhäuser, Boston, 1992.

3. Buser, P. and Silhol, R.: Geodesics, periods and equations of real hyperelliptic curves, Duke Math. J. 108 (2001), 211-250.

4. Näätänen, M. and Kuusalo, T.: On arithmetic genus 2 subgroups of triangle groups, Contemp. Math. 201 (1997), 21-28.

5. Nehari, Z.: Conformal Mapping, McGraw-Hill, New York, 1952.

6. Silhol, R.: Hyperbolic lego and algebraic curves in genus 2 and 3, Contemp. Math. 311 (2002), 313-334.

7. Silhol, R. and Buser, P.: http://www.math.univ-montp2.fr/ rs/genus2unifdoc.html.

8. Silverman, J.: The Arithmetic of EllipticCurves, Springer-Verlag New York; 1986. 